\title{
Detection and measurement of waviness on thin metallic wires
}

\author{
Cesar Tejeda, Luis Miguel Sanchez-Brea, and Eusebio Bernabeu
}

\begin{abstract}
We propose a model for determining the far-field diffraction pattern of wires with waviness. Analytical solutions are obtained by means of the stationary phase method, which allows us to determine dimensional parameters such as wire diameter and waviness factor. Experimental results are presented, which are in accordance with our theoretical description. (C) 2004 Optical Society of America

OCIS codes: $050.0050,120.4290,240.6700$.
\end{abstract}

\section{Introduction}

Metallic wires do not present an exact cylindrical shape, but surface structures may appear in their manufacture. ${ }^{1}$ These surface structures usually modify the physical-chemical properties of such wires, and their detection is therefore essential. Several of them, such as die lines, drawing grooves, and roughness, have already been analyzed from the point of view of detection, ${ }^{2-5}$ but there are important surface structures, such as waviness, that have not been examined. Waviness is a periodic deviation of the wire axis from linearity, and it is usually due to a defective or unsuitable straightening device (diameter of rolls, distance between rolls, or number of rolls) or a wrong roll arrangement in the manufacture line. ${ }^{6}$

Waviness is not always a surface structure to avoid. Currently, there is a need for the production of thin spiral wires, and industry is requesting systems for the determination of their diameter and other geometrical parameters, such as the waviness factor, which is the relationship between the amplitude and the pitch of waviness.

For straight wires, the measurement of the diameter is a well-known problem. The most common technique for thin-cylinder diameter estimation is based on the measurement of the distance between

The authors are with the Departamento de Óptica, Universidad Complutense de Madrid, Facultad de Ciencias Físicas, Ciudad Universitaria s/n 28040, Madrid, Spain. L. M. Sanchez-Brea's e-mail address is sanchezbrea@fis.ucm.es.

Received 2 August 2003; revised manuscript received 21 October 2003; accepted 28 October 2003.

0003-6935/04/071480-05\$15.00/0

(C) 2004 Optical Society of America two consecutive minima of the far-field diffraction pattern. ${ }^{7-9}$ However, it is not clear that such a model can be applied to wavy wires. Numerical solutions have been obtained for the problem of diffraction by wavy metal strips, ${ }^{10}$ although a simple analytical solution is in many cases more useful in that it provide us with formulas to determine the geometrical parameters.

In this paper we analyze, by determining the farfield diffraction pattern, how wavy wires (helicoidal and undulated) diffract light. In addition, we present a technique for estimating the diameter of wires with waviness, for detecting waviness, and for measuring the waviness factor.

\section{Diffraction of Helicoidal Wires}

One of the most important kinds of wire with waviness is the helicoidal wire [Fig. 1(a)]. To determine its diffraction pattern, we need to parameterize its surface. An helicoidal cylinder, with radius $R$, helix period $p$, and helix amplitude $r$, can be described as ${ }^{11}$

$$
\begin{aligned}
x= & r \cos (2 \pi \tau)-R[p v \sin (2 \pi \tau) \sin \chi \\
& +\cos (2 \pi \tau) \cos \chi], \\
y= & p \tau-2 \pi r R v \sin \chi, \\
z= & r \sin (2 \pi \tau)+R[2 \pi v \cos (2 \pi \tau) \sin \chi \\
& -\sin (2 \pi \tau) \cos \chi],
\end{aligned}
$$

where the axis of symmetry is oriented along the $y$ axis, $\chi, \tau$ are parameters that run from $[0,2 \pi)$ and $(-\infty, \infty)$, respectively, and $v=1 /\left(4 \pi^{2} r^{2}+p^{2}\right)^{1 / 2}$. To ensure that the surface does not intersect with itself, the condition $1 /\left(4 R^{2}\right)>1 / p^{2}+1 /\left(4 \pi^{2} r^{2}\right)$ must be fulfilled.

The simplest way to estimate the diffraction pat- 


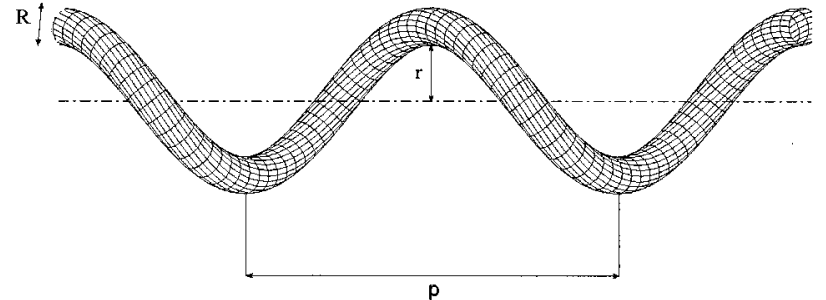

(a)

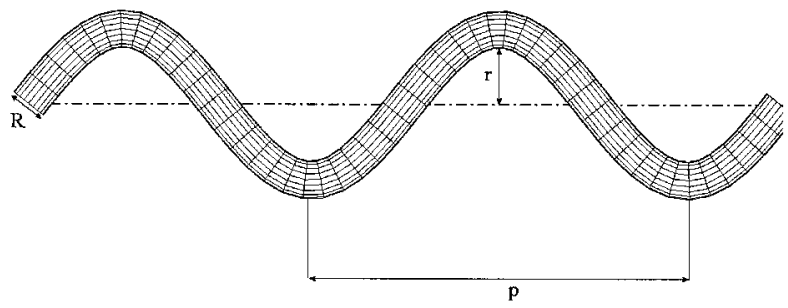

(b)

Fig. 1. Description of a (a) helicoidal wire and (b) undulated wire and parameters involved.

tern of the helicoidal wire in the far field is to approximate it with that of its projection. When $r, R \ll p$, which is the most common case, the projection of the helicoidal cylinder is approximately

$(x, y) \in$ wire surface $\Leftrightarrow$

$$
\Lambda(y)-\Gamma(y)<x<\Lambda(y)+\Gamma(y),
$$

where $[x, y]$ is the projection plane,

$$
\begin{aligned}
& \Lambda(y)=r \sin \left(\frac{2 \pi y}{p}\right), \\
& \Gamma(y)=R\left[1+\left(\frac{2 \pi r}{p}\right)^{2} \cos ^{2}\left(\frac{2 \pi y}{p}\right)\right]^{1 / 2} .
\end{aligned}
$$

According to the Fraunhofer approximation, the far-field diffraction pattern, assuming that the wire has infinite length, is

$$
\begin{aligned}
U(u, v)= & C k E_{0} \int_{-\infty}^{\infty} \int_{\Lambda(y)-\Gamma(y)}^{\Lambda(y)+\Gamma(y)} \exp [-i k \\
& \times(u x+v y)] \mathrm{d} x \mathrm{~d} y,
\end{aligned}
$$

where $C$ is a constant independent of the wavelength, $k$ is the wave number, and $u, v$ are coordinates representing the far-field direction.

\section{A. Numerical Analysis}

We have calculated numerically the solution to Eq. (3). The main features can be observed in Fig. 2: (a) Diffracted light is enclosed between two straight lines, (b) the maximum of intensity (masked in the figure) coincides with the incidence direction, (c) there are a series of diffraction minima that correspond to circles around the maximum of intensity, and (d) an extrastructure in the form of straight lines parallel to those that enclose diffracted light appears.

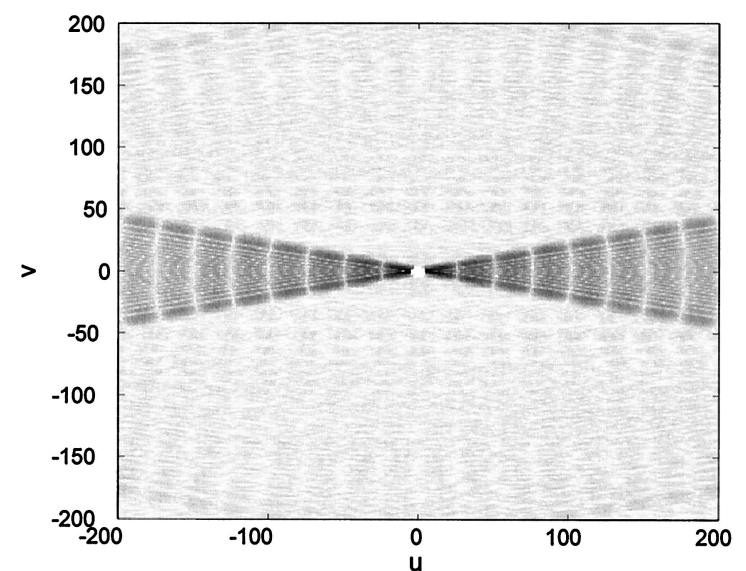

Fig. 2. Numerical simulation of the far-field diffraction pattern by use of Eq. (3) for helicoidal wire with parameters $R=50 \mu \mathrm{m}, r=$ $88 \mu \mathrm{m}$, and $p=2525 \mu \mathrm{m}$. The wavelength considered is $\lambda=$ $0.6328 \mu \mathrm{m}$. This diffraction pattern has been calculated by means of a fast-Fourier-transform algorithm.

Such features also appear in the experimental farfield diffraction of helicoidal wires (see Fig. 5 in Section 4), which confirms the validity of the assumption that the three-dimensional helicoidal wire behaves approximately as a thin aperture with the same projection.

\section{B. Phase Stationary Approximation}

A numerical analysis provides us with information of the diffraction pattern. However, our interest is to obtain a technique to determine geometrical parameters of helicoidal wires, and therefore an analytical solution is, in our case, more useful. For this, we will solve Eq. (3).

One can easily solve the integral in $\mathrm{d} x$, obtaining

$$
\begin{aligned}
U(u, v)= & \frac{2 C}{u} E_{0} \int_{-\infty}^{\infty} \sin [k \Gamma(y) u] \\
& \times \exp \{-i k[v y+u \Lambda(y)]\} \mathrm{d} y .
\end{aligned}
$$

However, the integral in $\mathrm{d} y$ cannot be solved exactly in the general case, and we have used an asymptotic approximation (the stationary phase method ${ }^{12}$ ). For this, we have written the integral as

$$
U(P)=\int_{-\infty}^{\infty} g(y) \exp [i k f(y)] \mathrm{d} y,
$$

where $g(y)=2 C E_{0} \sin [k \Gamma(y) u] / u$ and $f(y)=-[v y+$ $u \Lambda(y)]$. According to this method, when $k$ is large, the exponential term varies strongly, and positive and negative parts cancel each other everywhere except at points $y_{s}$, where $f(y)$ is stationary [d $f(y) /$ $\left.\left.\mathrm{d} y\right|_{y_{s}}=0\right]$. In this case, the integral is approximately

$$
\begin{aligned}
U(u, v) \approx & {\left[\frac{\pi}{k f^{\prime \prime}\left(y_{s}\right)}\right]^{1 / 2} g\left(y_{s}\right) \exp \left(i \left\{k f\left(y_{s}\right)\right.\right.} \\
& \left.\left.+\frac{\pi}{4}+\frac{\arg \left[f^{\prime \prime}\left(y_{s}\right)\right]}{2}\right\}\right),
\end{aligned}
$$


where $f^{\prime}$ represents the second derivative of $f$ with respect to $y$. After some calculations, we find that the stationary value is $y_{s}=p / 2 \pi \arccos (-v p / 2 \pi u r)$ and the field is

$$
\begin{aligned}
U(u, v) \approx & \frac{2 C E_{0} \exp \left\{i\left[k g\left(y_{s}\right)+\pi / 4\right]\right\}}{k^{1 / 2}}\left(\frac{p}{u}\right) \\
& \times \frac{\sin \left(k R \sqrt{u^{2}+v^{2}}\right)}{\left[(2 \pi r u)^{2}-(p v)^{2}\right]^{1 / 4}} .
\end{aligned}
$$

In that the output of the arccosine function is restricted to the interval $[-1,1]$, this solution is valid only at those locations where $-1<v p / 2 \pi u r<1$. Therefore light keeps inside the area enclosed by two straight lines in the $(u, v)$ plane: $u>-p v /(2 \pi r)$ and $u<p v /(2 \pi r)$. At the rest of locations the field $U(u$, $v)$ is zero. The intensity distribution in far field $I(u$, $v)=U(u, v) U^{*}(u, v)$ is then

$$
\begin{aligned}
I(u, v) \approx & \frac{4 C^{2} I_{0}}{k}\left(\frac{p}{u}\right)^{2} \frac{\sin ^{2}\left(k R \sqrt{u^{2}+v^{2}}\right)}{\left[(2 \pi r u)^{2}-(p v)^{2}\right]^{1 / 2}}, \\
& -1<v p / 2 \pi u r<1 \\
& =0 \text { elsewhere. }
\end{aligned}
$$

To have a better interpretation of the expression, let us change the coordinate system to polar: $u=\rho \cos$ $\varphi, v=\rho \sin \varphi$. Then expression (8) is expressed as

$$
\begin{aligned}
I(\rho, \varphi) \approx & \frac{4 C^{2} I_{0} p^{2} R^{2}}{\cos ^{2} \varphi\left[(2 \pi r \cos \varphi)^{2}-(p \sin \varphi)^{2}\right]^{1 / 2}} \\
& \times \frac{\operatorname{sinc}^{2}(k R \rho)}{\rho},
\end{aligned}
$$

where $\operatorname{sinc}(x)=\sin (x) / x$. We can see that the diffraction pattern for a wire with waviness presents a radial intensity distribution modulated according to a sinc function. In Fig. 3 the intensity distribution for several dimensional parameters is shown.

\section{Undulated Wires}

Undulations are also an important practical case in that they are normally considered a defective characteristic of metallic wires. Undulated wires present a periodic deviation of the wire axis in only one plane [Fig. 1(b)]. A wavy cylinder, with radius $R$, waviness period $p$, and amplitude $r$, can be described as ${ }^{11}$

$$
\begin{aligned}
& x=r \sin \left(\frac{2 \pi \tau}{p}\right)+\frac{R \sin \chi}{\left[1+(2 \pi r / p)^{2} \cos ^{2}(2 \pi \tau / p)\right]^{1 / 2}}, \\
& y=\tau-\frac{2 \pi r / p \cos (2 \pi \tau / p)}{\left[1+(2 \pi r / p)^{2} \cos ^{2}(2 \pi \tau / p)\right]^{1 / 2}} R \sin \chi \\
& z=R \cos \chi
\end{aligned}
$$

where the axis of symmetry is oriented along the $y$ axis and $\chi, \tau$ are parameters that run from $[0,2 \pi)$ and $(-\infty, \infty)$, respectively. To ensure that the surface does not intersect with itself, the condition $4 \pi^{2} R r<$

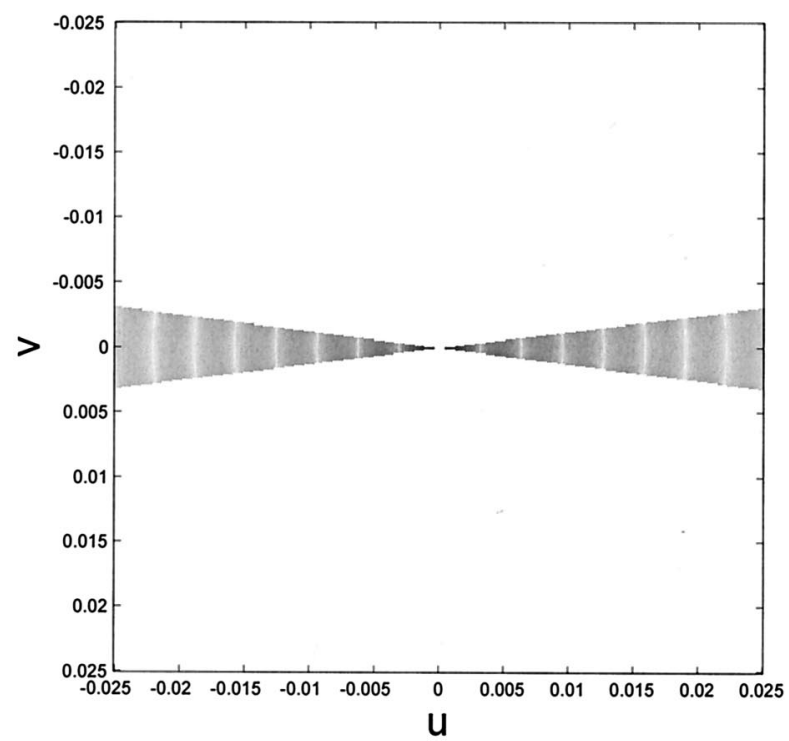

(a)

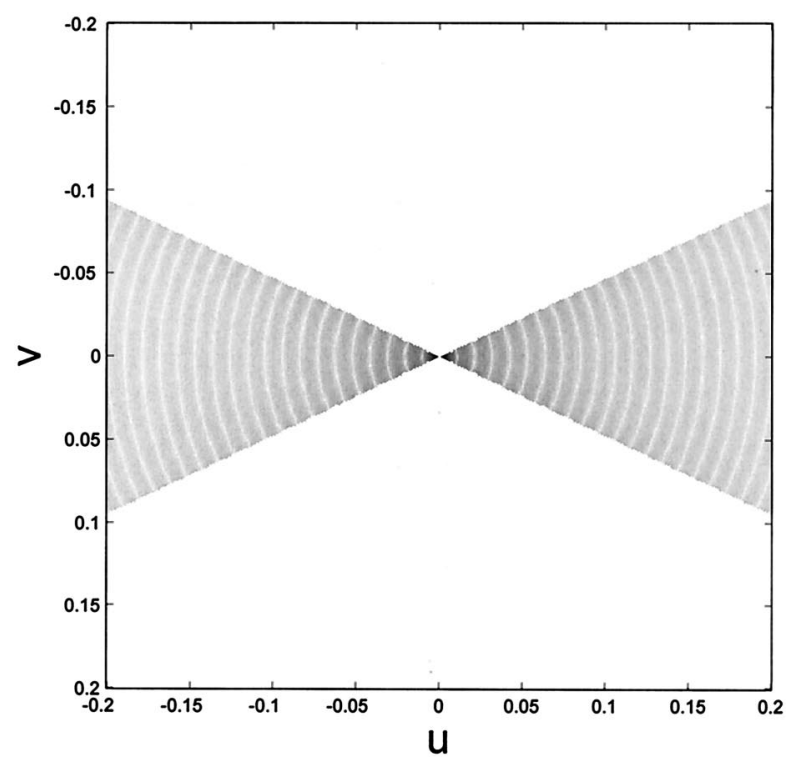

(b)

Fig. 3. Intensity distribution obtained by means of expression (8) for two wires with waviness (a) $r=40 \mu \mathrm{m}, p=2000 \mu \mathrm{m}$, and $R=$ $100 \mu \mathrm{m}$ and (b) $r=300 \mu \mathrm{m}, p=4000 \mu \mathrm{m}$, and $R=30 \mu \mathrm{m}$. In both cases the wavelength is $\lambda=0.6328 \mu \mathrm{m}$.

$p^{2}$ must be fulfilled. When waviness is small $(r, R$ $\ll p$ ), then the projection of the wavy cylinder is the same as that of the helicoidal wire [expression (2)], and the diffraction pattern for both geometries is the same, under the approximations considered.

\section{Parameter Estimation}

\section{A. Waviness Factor}

By means of expression (8) we can determine the relation $r / p$ between the period and the amplitude of waviness, also called the waviness factor. The denominator is real only at the locations where $(2 \pi r \cos$ 


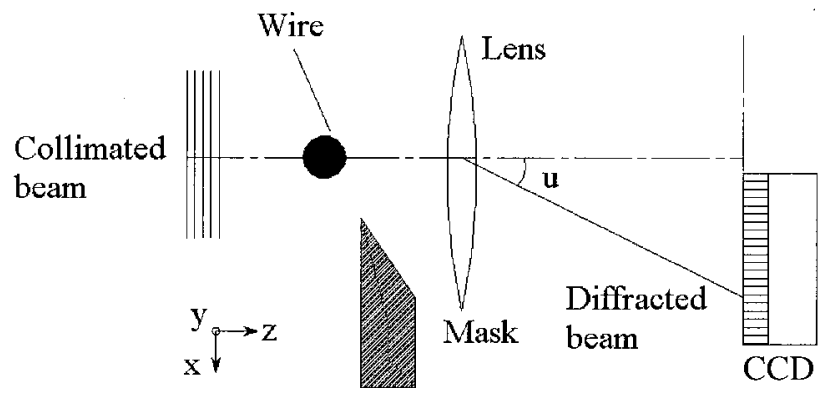

Fig. 4. Experimental setup for measuring the far-field diffraction pattern of wavy wires.

$\varphi)^{2}>(p \sin \varphi)^{2}$. At the rest of locations, there is not light at the diffraction pattern. This condition corresponds to two straight lines,

$$
\tan \varphi_{0}= \pm \frac{2 \pi r}{p}
$$

which generate the intensity cone (Fig. 3). As a consequence, by measuring these two slopes, it is possible to estimate the waviness factor.

\section{B. Diameter}

The usual way to determine the wire diameter is measuring the distance between two consecutive minima in the diffraction pattern. We can continue using the same technique, as is shown in expression (10). For example, the intensity at $\varphi=0$ is

$$
I(\rho, \varphi=0) \approx \frac{4 C^{2} p^{2} R^{2}}{k \rho} \frac{\operatorname{sinc}^{2}(k R \rho)}{2 \pi r} .
$$

To increase the output signal and therefore the signal-noise relationship, it is better to integrate expression (10) in the $\varphi$ coordinate. In such a case,

$$
\begin{aligned}
I(\rho) & =\int I(\rho, \varphi) \mathrm{d} \varphi \approx \frac{4 C^{2} p^{2} R^{2}}{\rho} \operatorname{sinc}^{2} \\
& \times(k R \rho) \int_{-\varphi_{0}}^{\varphi_{0}} \frac{1}{\cos ^{2} \varphi\left[(2 \pi r \cos \varphi)^{2}-(p \sin \varphi)^{2}\right]^{1 / 2}} \mathrm{~d} \varphi \\
& =8 C^{2} R^{2} p E\left[-\left(\frac{2 \pi r}{p}\right)^{2}\right] \frac{\operatorname{sinc}^{2}(k R \rho)}{\rho},
\end{aligned}
$$

where $E(x)$ is the complete elliptic integral of the second kind. In both cases, the location of minima are placed at zeros of the sinc function, i.e., $\rho_{m}=$ $m \lambda / R$ (where $m$ is an integer), and we obtain the wire radius by measuring the distances between two consecutive minima:

$$
R=\frac{\lambda}{\rho_{m}-\rho_{m-1}} .
$$

\section{Results}

We have measured the experimental far-field diffraction pattern for several wires with waviness. The setup (Fig. 4) consists of a typical Fraunhofer diffrac-

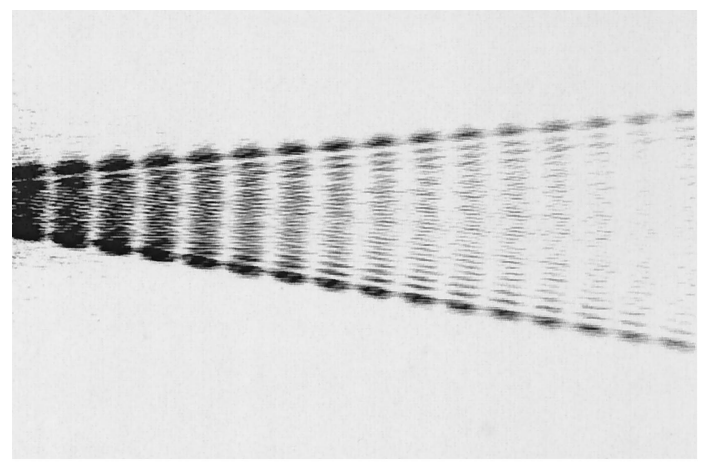

(a)

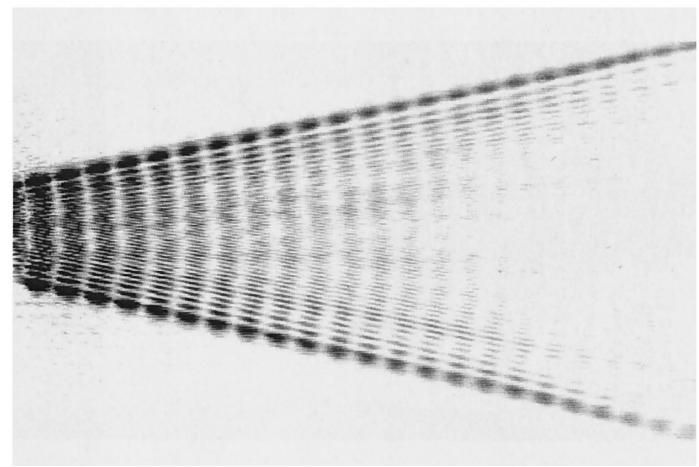

(b)

Fig. 5. Far-field diffraction patterns obtained for two wires with waviness: (a) $R=50 \mu \mathrm{m}, r=88 \mu \mathrm{m}$, and $p=2525 \mu \mathrm{m}$ and (b) $R=75 \mu \mathrm{m}, r=67 \mu \mathrm{m}$, and $p=2400 \mu \mathrm{m}$.

tion arrangement. The wire is illuminated with a collimated laser beam whose wavelength is $675 \mathrm{~nm}$. This laser beam is focused with a lens of $f=80 \mathrm{~mm}$, and it is collected at its focal plane with a bidimensional CCD, whose chip is an array of $768 \times 576$ pixels with a size of $8.8 \times 6.6 \mathrm{~mm}$. The CCD chip is not centered with respect to the optical axis; in contrast, it lays outside the axis so that the maximum at the center of the diffraction pattern cannot damage the camera. In addition, a knife stop right after the wire was inserted to eliminate undesired reflections of the beam.

With this setup, several diffraction patterns have been obtained for helicoidal and undulated wires with different diameters and waviness factors. In Fig. 5 a sample for two wires is displayed. For the wire used for Fig. $5(\mathrm{a}), R=50 \mu \mathrm{m}, r=88 \mu \mathrm{m}$, and $p=2525 \mu \mathrm{m}(r / p=0.035)$. For the wire used for Fig. 5(b), $R=75 \mu \mathrm{m}, r=67 \mu \mathrm{m}$, and $p=2400 \mu \mathrm{m}$ $(r / p=0.028)$. These parameters have been measured by optical microscopy (Leica microscope VMM200 with a QuadraCheck digital readout system).

With the proposed technique-Eqs. (15) and (12) the dimensional parameters for the wire used for Fig. 5 (a) are $R=48.9 \mu \mathrm{m}$ and $r / p=0.032$ and those for the wire used for Fig. 5(b) are $R=73.2 \mu \mathrm{m}$ and $r / p=$ 0.029 , which are close to the wire parameters measured. 
The experimental diffraction pattern, in addition to the radial geometry predicted by expression (10), presents a structure, close to the slopes, that is not explained with our approximation (see Fig. 5). Because such an extra structure appears in the numerical approach of Eq. (3), we think that the lack of such structure in our model is due to the asymptotic approximation and not to the calculus of field by use of the projection of the wire. Note that such a structure appears close to the caustics, and the stationary phase method fails at caustics, as Stamnes ${ }^{12}$ shows.

\section{Conclusion}

In this paper we analyze, by determining the far-field diffraction pattern, how helicoidal and undulated wires diffract light. Analytical solutions are obtained by means of the stationary phase method, which allows us to determine dimensional parameters, such as wire diameter and waviness factor. Experimental results are presented, which are in accordance with our theoretical description.

We thank Jose Alonso-Fernandez and Klaus Jakobs for their valuable suggestions and interest in this study.

This project was partially supported by the European Commission project SMT4-CT97-2184 Detection of Defects on Cylindrical Surfaces and by the Ministerio de Ciencia y Tecnología of Spain (contract DPI2001-1238). C. Tejeda was supported by a Marie Curie Research Training Grant (contract SMT4-CT98-9028). Part of this research was carried out at KJM Gesellschaft für Optoelektronische Messtechnik mbH (Germany).

\section{References}

1. E. Bernabeu, L. M. Sanchez-Brea, P. Siegmann, J. A. GomezPedrero, G. Wilkening, L. Koenders, F. Müller, M. Hildebrand, and H. Hermann, "Classification of surface structures on fine and ultra fine wires,” Appl. Surf. Sci. 180, 191-199 (2001).
2. L. M. Sanchez-Brea, P. Siegmann, M. A. Rebollo, and E. Bernabeu, "Optical technique for the automatic detection and measurement of surface defects on thin metallic wires," Appl. Opt. 39, 539-545 (2000).

3. L. M. Sanchez-Brea, P. Siegmann, E. Bernabeu, M. A. Rebollo, F. Pérez-Quintián, and C. A. Raffo, "Detection of surface defects on thin metallic wires by geometrical conical refraction," Wire J. Int. 33, 124-127 (2000).

4. J. C. Martínez-Antón, P. Siegmann, L. M. Sanchez Brea, and E. Bernabeu, "In-line detection and evaluation of surface defects on thin metallic wires," in Optical Measurement Systems for Industrial Inspection II: Applications in Production Engineering, R. Hoefling and W. P. Jueptner, eds., Proc. SPIE 4399, 27-31 (2001).

5. R. Berlasso, F. Perez-Quintián, M. Rebollo, N. Gaggioli, L. M. Sanchez-Brea, and E. Bernabeu, "Speckle size of light scattered from slightly rough cylindrical surfaces," Appl. Opt. 41, 2020-2027 (2002).

6. E. Bernabeu, L. M. Sanchez-Brea, P. Siegmann, J. A. GomezPedrero, G. Wilkening, L. Koenders, F. Müller, M. Hildebrand, and H. Hermann, Surface Structures on Fine and Ultra Fine Wires (Editorial Complutense, Madrid, 2002).

7. D. Lebrun, S. Belaid, C. Ozkul, K. F. Ren, and G. Grehan, "Enhancement of wire diameter measurements: comparison between Fraunhofer diffraction and Lorenz-Mie theory," Opt. Eng. 35, 946-950 (1996).

8. E. Bernabeu, I. Serroukh, and L. M. Sanchez-Brea, "Geometrical model for wire optical diffraction selected by experimental statistical analysis," Opt. Eng. 38, 1319-1325 (1999).

9. J. C. Martínez-Antón, I. Serroukh, and E. Bernabeu, "On Babinet's principle and a diffraction-interferometric technique to determine the diameter of cylindrical wires," Metrologia 38, 125-134 (2001).

10. S. L. Prosvirnin, S. A. Tretyakov, and P. L. Mladyonov, "Electromagnetic wave diffraction by planar periodic gratings of wavy metal strips," J. Electromagn. Waves Appl. 16, 421-435 (2002).

11. E. Kreyszig, Differential Geometry (Dover, New York, 1991), pp. $24-25$.

12. J. J. Stamnes, Waves in Focal Regions (Adam Hilger, Bristol, 1986) 